\title{
Radiatively driven Rayleigh-Taylor instability candidates around a forming massive star system
}

\section{NACO adaptive optics and VISIR study of G333.6-0.2}

\begin{abstract}
M. S. N. Kumar
Centro de Astrofisica da Universidade do Porto, Rua das estrelas, 4150-762 Porto, Portugal e-mail: nanda@astro.up.pt

Received 2 April 2013 / Accepted 5 August 2013

\section{ABSTRACT}

The formation of the highest mass stars are thought to be dominated by instabilities resulting from gravitation and radiation. Instabilities due to gravitation are commonly demonstrated by observations of fragmentation, but those due to effects of radiation have thus far not been found. Here I report on the NACO adaptive optics and mid-infrared diffraction-limited VISIR imaging data of an extemely luminous ultra-compact HII region G333.6-0.2. Two infrared sources, one bright in the near-infrared (appearing pointlike) and another in the mid-infrared (resolved with an elliptical shape) are uncovered through this data, which are located at the heart of this region. These infrared sources appear to be embedded in the waist of a bipolar-shaped nebula and UCHII region, the lobes of which are separated by a dark patch. Dense filamentary features with finger/hook morphology are found; they appear to be connected to the two bright infrared sources and the sizes of these hook features are sharply limited to $<5000$ AU. The observed properties of this target and a large amount of previous data obtained from the literature are compared together with the results of various numerical simulations of high-mass star formation. This comparison favours the interpretation that the finger/hook-like structures likely represent radiatively driven Rayleigh-Taylor instabilities arising in the outflow cavity of a forming high-mass binary star system.
\end{abstract}

Key words. stars: formation - stars: massive - HII regions

\section{Introduction}

The formation of the most massive stars in our Galaxy is an enigmatic topic in astrophysics revolving around a singular problem: the dissipation of intense radiation pressure in the early stages of massive protostellar collapse, which is thought to be capable of reversing accretion flows (see Zinnecker \& Yorke 2007). The work-around solution to this problem suggested by theory argues that stars as massive as $140 M_{\odot}$ can form through disk-mediated accretion (Nakano 1989; Yorke \& Sonnhalter 2002; Krumholz et al. 2009; Kuiper et al. 2010) because radiation is dissipated through outflow cavities. In recent years, detailed numerical simulations of massive star formation have been carried out; the majority of these are radiation-hydrodynamic (Krumholz et al. 2009; Kuiper et al. 2010, 2011; Cunningham et al. 2011; Peters et al. 2010a,b), with an increasing focus on magneto-hydrodynamic simulations (Hennebelle et al. 2011; Myers et al. 2013). Each of these simulations varies in its initial conditions and focus, leading to different approximations and limitations impacting the way in which realistic systems are represented. Most importantly, simulations using adaptive mesh refinement codes together with non-axisymmetric geometries (e.g. Krumholz et al. 2009; Hennebelle et al. 2011; Peters et al. 2010a) produce distinct fine structures at physical scales of a few hundred to a thousand AU.

For example, radiation hydrodynamic simulations of Krumholz et al. (2009, hereafter KKM09), and Kuiper et al. (2010, 2011) show distinctly different features due to such treatment. While the radiation-pressure-driven bubble rises along the outflow axis in both cases, KKM09 simulations produced
Rayleigh-Taylor instabilities (RTI) in the outflow bubble where radiation itself behaves like a lighter fluid penetrating into the denser gaseous matter (heavy fluid) surrounding the star. The optically thin gaps in between the RTI allowed radiation to escape, and the overdense material falls back to the disk plane. On largers scales, infalling gas is thought to strike the walls of the outflow cavity bubble, which effectively slides and settles down to the disk plane. The simulations of KKM09 also amplify the gravitational instabilities built up due to the swing mechanism (Adams et al. 1989), resulting in two massive stars. However, they used grey flux-limited diffusion (FLD) radiative transfer without including the direct radiation pressure. On the other hand, although Kuiper et al. (2010, 2011) used frequencydependent ray-tracing for direct radiation they did not produce RTI features and thus eventually questioned their stability as discussed in Kuiper et al. (2012). The topic of observational investigation in this work is therefore centred on the role of RTI in the mechanism of high-mass star formation.

Observations of ultra-compact HII regions at sub-arcsecond resolutions (Wood \& Churchwell 1989; Kurtz et al. 1994; Urquhart et al. 2009), mostly from the Very Large Array, have thus far not uncovered significant sub-structures at a level of a few hundred to a thousand AU. For instance, adaptive optics observations with the $8.2 \mathrm{~m}$ Very Large Telescope (VLT) of the UCHII region G5.89-0.39 (Feldt et al. 2003) in the $K_{\mathrm{s}}$ and $L^{\prime}$ bands reveal structure at such small scales. However, those observations were obtained much before recent theoretical developments to allow direct comparison. Finding physical structures resulting from instabilities associated with high-mass star formation depends not only on high-quality observations but 
also on favourable targets that satisfy the criteria of being nearby, young, and extraordinarily luminous. Massive (OB-type) stars are very few, representing just over $0.1 \%$ of all main-sequence stars in our Galaxy. Thus, stringent conditions on observations and target selection exist in order to verify the validity of theoretical scenarios.

The UCHII region G333.6-0.2 is known to possess some extraordinary qualities and is situated inside the giant HII region complex RCW106, which represents parts of a long filamentary giant molecular cloud (Mookerjea et al. 2004). Soon after the advent of far-infrared astronomy in the late 1960s, it was realized that this is a powerful source (Becklin et al. 1973), comparable with the Galactic centre and the famous stellar beast, Eta Carinae (Sutton et al. 1974). Located at a distance of 3.1-3.6 kpc to Sun, it has a bolometric luminosity of $3 \times 10^{6} L_{\odot}$ and is diagnosed as being compact (11" diameter) and dusty, as well as hosting highly ionizing stars (Becklin et al. 1973; Natta \& Oliva 1985). A distance of $3.6 \mathrm{kpc}$ will be adopted in this work to allow conservative interpretation of the specific results here. The observed Lyman continuum flux (LyC) from this compact region is estimated to be $N(\mathrm{LC}) \sim 1 \times 10^{50} \mathrm{~s}^{-1}$, which is equivalent to that emitted by about 19 O7V stars (Fujiyoshi et al. 2006). The UCHII region itself is embedded in a massive molecular clump of $\sim 15000 M_{\odot}$ (Mookerjea et al. 2004) from which a forest of emission lines tracing dense gas is detected (Lo et al. 2009). The dense clump hosts an embedded cluster, but the infrared and radio peaks within the target are coincident and compact. At sub-arsec resolution, this compact peak reveals itself as a dark patch in the near-infrared $K$ band, which forms the waist of a bipolar-shaped UCHII region and infrared nebula. The dark patch is suggestive of a large toroidal structure, and the bipolar shaped HII region and infrared nebula likely represent a radiation-driven outflow bubble (see Fig. 1). Here I present adaptive optics observations of this compact region and analyse it using various infrared and radio data at (sub)arcsecond resolutions. This exercise has resulted in the discovery of small-scale density structures $\left(<1^{\prime \prime}\right)$ with filamentary and finger/hook-like morphology associated with two luminous sources that appear point-like at $0.1^{\prime \prime}-0.3^{\prime \prime}$ level. These data are used to confront some outcomes produced by various numerical simulations.

\section{Observations and data reduction}

Data used in this work are extracted and processed from various archives.

Infrared observations: Near-infrared adaptive optics imaging data in the $J, H, K_{\mathrm{s}}, \mathrm{Br} \gamma(\mathrm{NB} 2.17)$, and $L^{\prime}$ bands were obtained using the NAOS-CONICA (NACO) system, which is mounted on the European Southern Observatory (ESO) $8.2 \mathrm{~m}$ VLT Yepun. These data correspond to the proposal ID: 075.C0424(A)(PI: Schödel). Mid-infrared $12 \mu \mathrm{m}$ and $18 \mu \mathrm{m}$ imaging observations were made using the VISIR/VLT (ESO proposal ID: 60.A-9234(A) PI: Siebernmorgen). A broad-band $K_{\mathrm{S}}$ band image from the VISTA Variables in the Via-Lactea (VVV) survey (Minniti et al. 2010) was retrieved to calibrate astrometry and photometry of the adaptive optics data. The spatial resolution achieved with NACO is highest for the $L^{\prime}$ band at $0.1^{\prime \prime}$, slightly lower in the $J, H, K_{\mathrm{s}}$, and $\mathrm{Br} \gamma$ bands between $0.1^{\prime \prime}-0.2^{\prime \prime}$, and diffraction limited at $0.3^{\prime \prime}$ and $0.5^{\prime \prime}$ in the $12 \mu \mathrm{m}$ and $18 \mu \mathrm{m}$ bands respectively.

Individual frames were recalibrated and combined to produce mosaics in each band. Astrometric calibration for images from $J$ to $L^{\prime}$ band was achieved by a triangulation method using the position of all stars in the NACO images against 2MASS

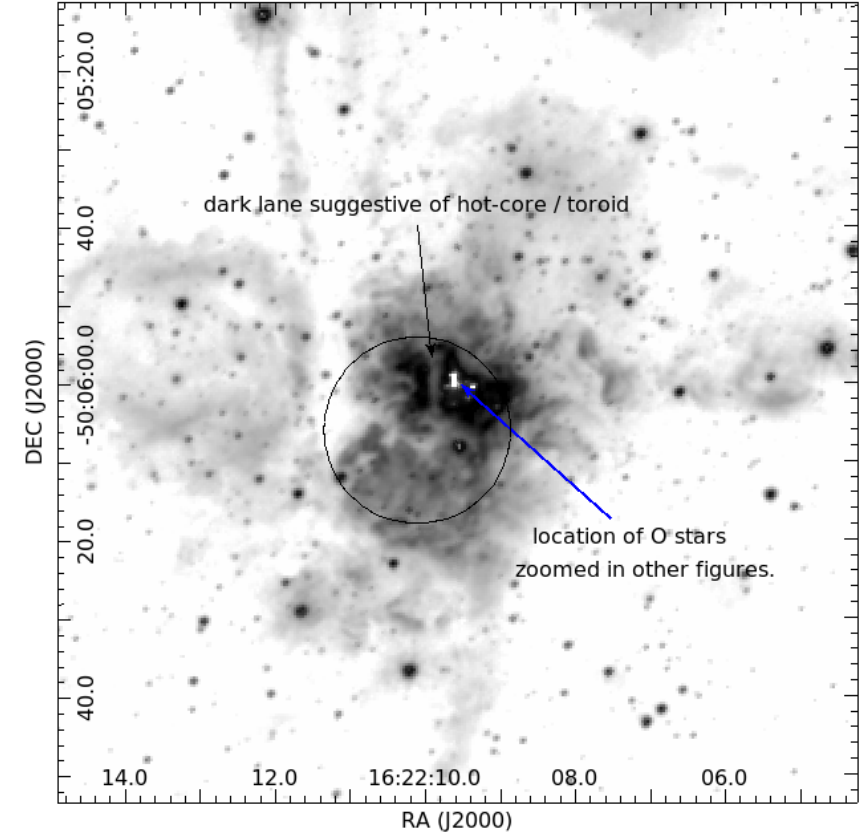

Fig. 1. A near-infrared $K$ s-band image of the source from the VVV survey $\left(0.5^{\prime \prime}\right.$ resolution $)$ is shown using inverted grey scale and logarithmic intensity stretch. The circle marks the $24^{\prime \prime}$ beam of the $1.2 \mathrm{~mm}$ observations that uncovered the cold and dense $\left(n_{\mathrm{H}_{2}}=5.44 \times 10^{4} \mathrm{~cm}^{-3}\right)$ molecular clump (Mookerjea et al. 2004), with an estimated mass of $\sim 15000 M_{\odot}$ and a radius of $1.88 \mathrm{pc}$. Intense line emission from a variety of molecular species $\left(\mathrm{CO}, \mathrm{CS}, \mathrm{HCO}^{+}, \mathrm{HCN}, \mathrm{HNC}, \mathrm{C}_{2} \mathrm{H}\right)$ is detected from this clump (Lo et al. 2009). The bipolar-shaped nebula and the dark lane are identical in morphology to the UCHII region (Fujiyoshi et al. 2006). The dark lane (size $\sim 30000 \mathrm{AU}$ ) may represent a hot core/toroid seen edge-on, but further analysis here indicates that it may actually represent the low-density part of the red-shifted outflow cavity (see Sect. 5).

positions. On the VISIR images, the target appears mostly extended, without any well-defined point source. Therefore we applied a cross-correlation of the nebular features between VISIR images and calibrated the NACO $L^{\prime}$-band image. The final astrometric accuracy is, therefore, limited by the 2MASS accuracy of $\sim 0.1^{\prime \prime}$. Aperture photometry was performed for selected sources using apertures of $0.5^{\prime \prime}$. Photometric zero-points measured at the VLT were applied first. Subsequent improvement was achieved by cross-calibrating the first pass photometry with that of VISTA photometry. Since the VISTA photometry is tied to the 2MASS photometry using several hundred stars in a given frame, it is robust with conservative maximum errors of $10 \%$.

Chandra X-ray data analysis: Chandra X-ray telescope observations of this target were obtained through observation ID:9911 (PI: L. Townsley). The data were reprocessed using CIAO software, following the analysis threads on the Chandra website to produce event files. The final goal is to evaluate the pressure from shocked gas resulting from stellar winds (Sect. 3.1). A background-subtracted spectrum was extracted for a region covering the features of interest without including point sources (stars). The background region was chosen about $4^{\prime}$ away from the G333.6-0.2 UCHII region. The spectrum was fitted using two XSPEC components (phabs and apec). A statistically good fit was obtained with a reduced chi-squared value of 0.99 , with 10 degrees of freedom. The resulting fit and the residuals are shown in Fig. 2. For the phabs model, we began with an initial guess of the line-of-sight hydrogen column density of $2.17 \times 10^{22} \mathrm{~cm}^{-2}$. However, the modelling results in a 


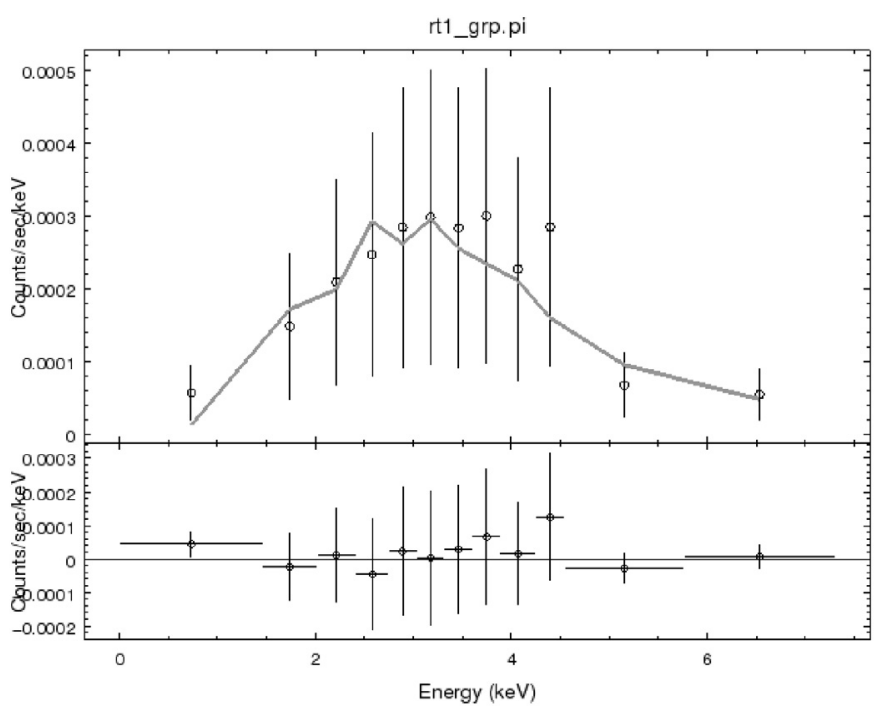

Fig. 2. Chandra X-ray spectrum analysis. The top panel shows the $X$-ray spectrum for the region, including the IRS 1 and IRS 2 sources. The solid grey line shows the fitted XSPEC model with phabs and apec components. The bottom panel displays the residuals after fitting.

final value of $n_{\mathrm{H}}=4.46 \times 10^{22} \mathrm{~cm}^{-2}$; this is a consistent value, considering that the UCHII region is embedded inside a dense and massive molecular clump. The APEC model component resulted in an X-ray temperature of $k T_{\mathrm{X}}=2.48 \mathrm{keV}$. The emission measure was obtained using the normalization parameter from the APEC model: norm $=\frac{E M \times 10^{-14}}{4 \pi D^{2}}$, where EM is the emission measure, $D$ is the distance to the source. Then the number density $n_{\mathrm{X}}$ was calculated using $n_{\mathrm{X}}=\sqrt{\frac{E M}{V}}$, where $V$ is the volume of the region as measured from the region file used to extract the X-ray spectrum. The size of the UCHII region $\left(10^{\prime \prime}\right)$ is considered to be the diameter of a sphere in computing the volume, and a distance of $3.6 \mathrm{kpc}$ to the target is assumed.

\section{Characteristics of the G333.6-0.2 region}

A near-infrared $K_{\mathrm{s}}$-band view of the G333.6-0.2 region can be found in Fig. 1. At a seeing of $0.5^{\prime \prime}$, this image is an improvement over similar images obtained with a $4 \mathrm{~m}$ class telescope described by Fujiyoshi et al. (2005). Mapped at the resolution of an arcsecond (Fujiyoshi et al. 2006), the UCHII region is fairly accurately represented by the bright near-infrared bipolarshaped nebula, separated by a dark patch seen in Fig. 1. The dark patches delineating the midriff of bipolar-shaped UCHII regions may represent large molecular toroids, a classical example being S106 region in the Cygnus region. High-resolution $1.3 \mathrm{~cm}$ mapping of S106 region reveals a well-defined bipolar-shaped HII region (Felli et al. 1984). The optical and near-infrared dark patch at the midriff of the S106 compact HII region is found to represent a large toroid traced by $2 \mathrm{~mm}$ dust continuum emission (Furuya et al. 2002). Similarly, the dark patch here in G333.6-0.2 may represent a large toroid; uncovering it will require high-spatial-resolution observations with appropriate molecular-line and dust tracers.

The margin of the western lobe of the bipolar nebula in G333.6-0.2 is so bright that it appears saturated in most infrared images obtained even with moderate facilities. This is because two ionizing sources are located at this margin (and at the edge of the dark patch): one bright at mid-infrared and the other at near-infrared wavelengths. They are denoted as IRS 1 and IRS 2 (see Figs. 3 and 4a). The brighter of the two peaks at longer wavelengths, IRS 1 becomes increasingly bright from $12 \mu \mathrm{m}$ to $18 \mu \mathrm{m}$, resolving out as a clear elliptical structure in the $18 \mu \mathrm{m}$ image (blue dashed contours in Fig. 4a). This elliptical structure represent polycyclic aromatic hydrocarbon and warm dust emission arising from a circumstellar disk/envelope. The NACO-AO data shows that IRS 1 is invisible at shorter wavelengths, whereas IRS 2 appears as a star. Indeed, the $18 \mu \mathrm{m}$ emission surrounds IRS 2 rather than peaking on it. The low-intensity peak coincides with a small dark lane to the northwest of IRS 2, as can be seen from the overlays in Fig. 4a. The sources IRS 1 and IRS 2 therefore appear to be embedded in a single, large toroidal structure of $\sim 5000 \mathrm{AU}$ in diameter seen edge-on. The $J, H$, and $K_{\mathrm{S}}$ magnitudes of IRS 2 are 13.1, 11.7 and 10.2 respectively. The faint near-infrared star close to IRS 1 (Fig. 4a) is a reddened object without any infrared excess, the nature of which is difficult to classify in the absence of an accurate extinction measurement of the region probed. The $10 \%$ accuracy of adaptive optics photometry is insufficient to understand the accurate nature of these sources, particularly in this heavily extincted region. The UCHII region itself is modelled to represent a champagne-flow-like outflow cavity bubble expanding in a perpendicular direction to the plane of this toroidal structure hosting the two sources (Fujiyoshi et al. 2006). The ionised outflow cavity is traced and characterised by the $\mathrm{H} 90 \alpha$ radio recombination and centimeter continuum imaging at 1.6" resolution (Fujiyoshi et al. 2006).

\subsection{Filaments and finger/hook like features}

From Figs. 3 and $4 \mathrm{a}$ it can be seen that tiny filamentary features with finger/hook morphology are found along the proposed outflow cavity. We note that they appear prominently amidst bright nebular emission, which is likely swamped by reflected light. At a spatial resolution approximately ten times better, these features represent details of previously known morphology (Fujiyoshi et al. 2001). The $8-12 \mu \mathrm{m}$ spectro-polarimetric studies have been used to show that the emission in this region is composed of both absorptive and emissive polarized components (Fujiyoshi et al. 2001). Also, significant line emission in the $\mathrm{Br} \gamma$ and $12.8 \mu \mathrm{m}$ [NeII] emission originates from this compact region (Fujiyoshi et al. 1998, 2006; Grave et al. 2013). Therefore the filamentary features are very likely tracing layers of hot ionized gas and/or dust with predominant small particle emission. Given the high temperatures in the close vicinity of the luminous sources, larger grains are typically destroyed. Similarly, molecular gas should be completely absent by exclusion effect of the above primary composition; this can be justified to a certain degree by the lack of $\mathrm{H}_{2}$ emission in this region (Moneti \& Moorwood 1989; Fujiyoshi et al. 2006). It is to be noted that the existing simple narrow-band imaging failed to produce discernable features because continuum subtraction is very uncertain and not effective in a region such as this without spectral imaging. Nevertheless, indications for such properties can be visualised through a colour composite, as shown in Fig. 4 a. Because the $L^{\prime}-, \mathrm{Br} \gamma$-, and $H$-band images used in the colour composite are all scaled similarly, the red and green colours represent relatively denser and more ionised parts. These spatially resolved features are limited to $<5000 \mathrm{AU}$ in length. Such small-scale filamentary loops have not been found in surveys of UCHII regions or compact nebulae. The length and width of the most prominent filaments are measured on the $L^{\prime}$ image at various positions. The lengths are measured using a straight line and the widths by using intensity profile cuts at multiple positions 

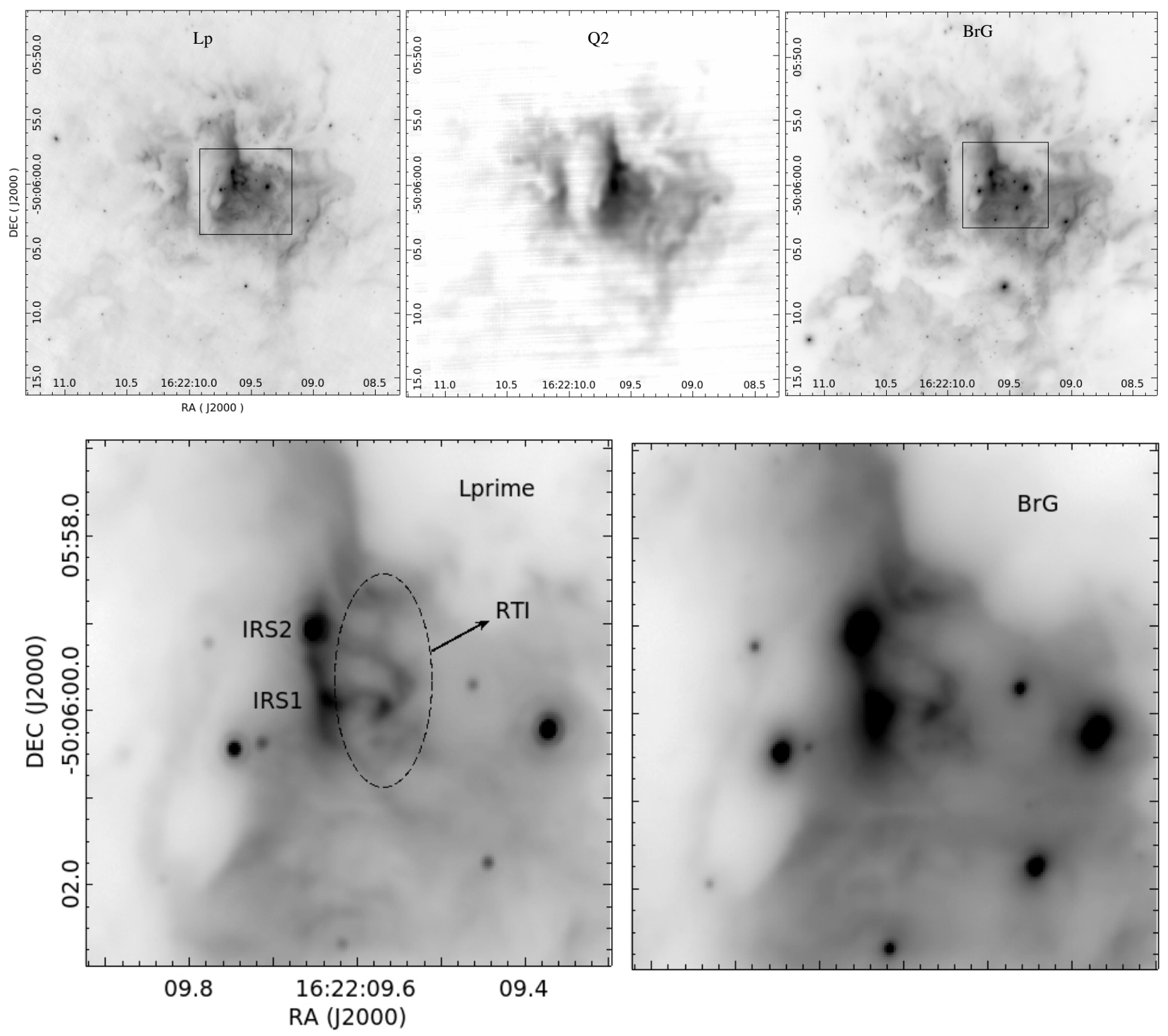

Fig. 3. Top panel: the NACO and VISIR data sample in the $L^{\prime}, Q 2$, and $\mathrm{Br} \gamma$ bands of the G333.6-0.2 UCHII region is displayed using a logarithmic inverted grey scale. The images display noticeable differences from the near-infrared to mid-infrared, which can be better visualized from Fig. 4a. Bottom panel: a zoom-in view of the central region (marked with a box in the top panel) displayed using square root scaling to show the IRS sources and RTI features clearly.

across the filaments. These measurements result in mean values of $0.63^{\prime \prime} \pm 0.29^{\prime \prime}$ and $0.16^{\prime \prime} \pm 0.03^{\prime \prime}$ for the lengths and widths of the filaments respectively. At a distance of $3.6 \mathrm{kpc}$, this estimate translates to a mean length of $2268 \pm 1044 \mathrm{AU}$ and mean width of $576 \pm 108 \mathrm{AU}$. The observed widths $(\sim 500 \mathrm{AU})$ are mostly an effect of the $0.1^{\prime \prime}$ spatial resolution from the adaptive optics images. The longest filament is the one associated with IRS 2 source measuring $~ 4000 \mathrm{AU}$ in length. Filamentary structures in the interstellar medium (ISM) are known to be ubiquitous in the Galaxy and are found in all molecular clouds. The ISM filaments are much larger in size: spatially resolved characterization using HERSCHEL data estimate their widths to be $0.1 \pm 0.03 \mathrm{pc}$ $\sim 20000 \mathrm{AU}$ (Arzoumanian et al. 2011), which is a factor of four larger than the lengths of the features found here.

\section{Radiation Rayleigh-Taylor instabilities?}

The filamentary features found above were exhaustively compared with the outcome of numerical simulations from KKM09,
Cunningham et al. (2011), Hennebelle et al. (2011), and Peters et al. (2010a,b, 2011), which have produced structures at a few hundred to a few thousand AU. Apart from comparing the morphology, the observed properties in the infrared to radio are analysed and supplemented by simple calculations. Towards the end of this section, it becomes apparent that the net result of this analysis suggests that the observed filamentary features likely represent RTI features residing in the ionized outflow cavity of G333.6-0.2. Indeed, as shown in Figs. 4a and b, the core of G333.6-0.2 displays good resemblance with a snapshot of the edge-on view of the simulations by KKM09. In the following, I present evidence to show that the observed resemblance between data and simulation snapshot is more than simple coincidence.

To begin, I recall the salient characteristics of the substructures produced by the simulations under consideration: a) filaments due to RTI prominently appear in the outflow cavity in the simulations of KKM09 and are essentially absent elsewhere, b) filaments produced by Hennebelle et al. (2011) are 


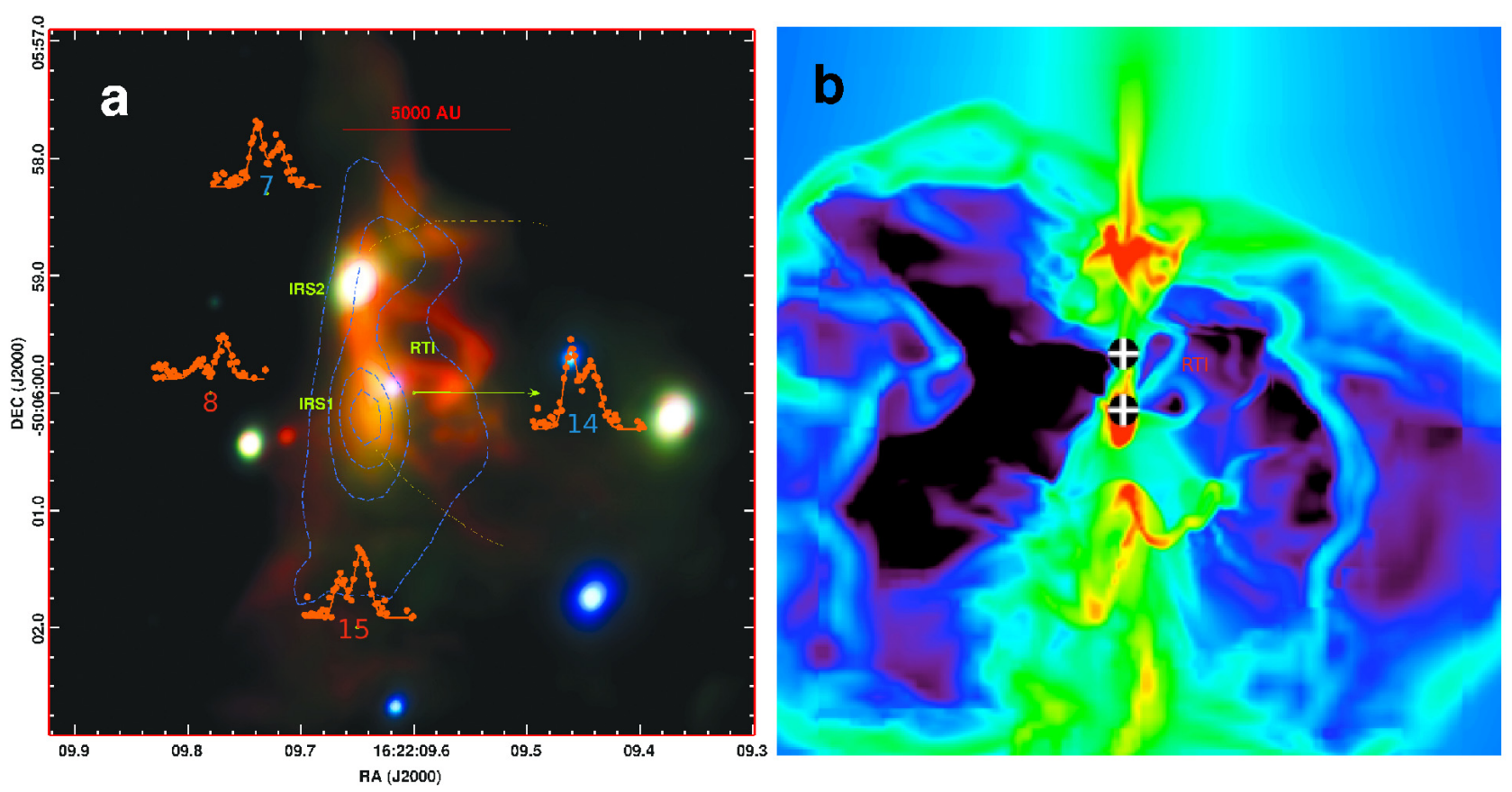

Fig. 4. a) Near-infrared $H$-, Br $\gamma$-, and $L^{\prime}$-band adaptive optics data coded in blue, green, and red respectively to produce the colour composite of the brightest part of the ultra-compact HII region G333.6-0.2. Blue dashed contours depict $18 \mu \mathrm{m}$ emission revealing the source IRS 1. H90 $\alpha$ recombination-line double-peaked spectra from Fujiyoshi et al. (2006) are overplotted. The numbers below these spectra correspond to the numbering in the original paper (see their Figs. 4 and 5). The thin orange line sketch represents possible margin of the outflow bubble. b) Simulations of a forming massive star system from Krumholz et al. (2009) showing $3000 \mathrm{AU} \times 3000 \mathrm{AU}$ region at 41700 years, where the colours represent volume densities. The disk plane and outflow axis are aligned vertically and horizontally.

radially oriented, and appear in all three axes $(x, y, z)$ representing magnetised ropes of material converging to a centre, and c) simulations of Peters et al. (2010a,b, 2011) result in small structures caused by the pressure of photoionized gas, which typically arises outside a region where the escape speed is $>10 \mathrm{~km} \mathrm{~s}^{-1}$.

Figure 4a displays two salient features: a) filamentary features with finger/hook morphology that are sharply limited in size to $\leq 1.3^{\prime \prime}$ and also appear connected to IRS 1 and IRS 2, b) sources IRS 1 and IRS 2 appear similar in mass, despite their different infrared properties because the temperature estimate is roughly the same for both sources at $>40000 \mathrm{~K}$ (see Grave et al. 2013).

As evidenced from NACO data in Figs. 3 and 4a, the features in question are restricted to a small space around the forming stars and limited in lengths, preferentially found within the previously known outflow cavity. Therefore they are unlike the radially converging magnetised rope-like filaments from Hennebelle et al. (2011). Next, the short lengths and visibly linked nature to the IRS sources show that they arise from within a region where the escape speeds are greater than $10 \mathrm{~km} \mathrm{~s}^{-1}$ (see below). The smallest and brightest finger/hook-like feature is $2000 \mathrm{AU}$ in length and appears connected to the IRS sources. The escape velocity for photoionised gas at this distance is $\sim 8 \mathrm{~km} \mathrm{~s}^{-1}$, considering a star of $40 M_{\odot}$ surrounded by a disk of $20 M_{\odot}$. In reality, the gravitational pull will be much larger because: a) together, IRS 1 and IRS 2 can contribute a mass $\sim 100 M_{\odot}$ or more, and b) the disk/envelope plane will contain an even larger parcel of mass. This assumption is made considering the elliptically shaped hot dust emission at $18 \mu \mathrm{m}$, which is primarily due to small-grain dust (micron sized) and also the spatial coincidence with the $1.2 \mathrm{~mm}$ peak representing the massive and dense clump (15000 $M_{\odot}$ and $n_{\mathrm{H}_{2}}=5.44 \times 10^{4} \mathrm{~cm}^{-3}$; Mookerjea et al. 2004). Also, the observed features are not due to hot gas expanding at the sound speed of $\sim 10 \mathrm{~km} \mathrm{~s}^{-1}$. This is because the sound speed and the escape velocity are comparable in magnitude, making it improbable that hot gas pressure drives the 2000-3000 AU large observed features. Indeed, as elaborated in Sect. 5 , the features coincide with a region where double-peaked H90 $\alpha$ recombination lines clearly display peak-to-peak velocities of $20-30 \mathrm{~km} \mathrm{~s}^{-1}$. These double peaks are resolved components located on opposite sides with respect to the ambient velocity, which is found to be $V_{\mathrm{LSR}}=-47 \mathrm{~km} \mathrm{~s}^{-1}$ by lower spatial resolution observations (McGee et al. 1975). Together, these considerations suggest that the observed features are unlikely to be those arising in the simulations by Peters et al. (2010a,b, 2011) and are also not due to hot gas expanding at the sound speed of $\sim 10 \mathrm{~km} \mathrm{~s}^{-1}$.

Instead, the observed features satisfy many qualities expected from RTI structures produced by KKM09, as discussed below. The first requirement for the development of RTI is that radiation (isotropic) should be strong enough to imitate a light fluid capable of upholding a denser overlying fluid. The two sources IRS 1 and IRS 2 are the only resolved ones that coincide with the peak of the UCHII region G333.6-0.2, which is known to emit LyC photons equivalent to that of 19 O7V stars. These sources are $\sim 1^{\prime \prime}$ apart, corresponding to $\sim 3600$ AU at a distance of $3.6 \mathrm{kpc}$. Mid-infrared spectra obtained with the VISIR instrument on VLT at a similar spatial resolution of $0.3^{\prime \prime}$ by placing the slit along IRS 1 and IRS 2 have uncovered intense emission of [ArIII] and [NeII] lines, which localise and peak (within $0.3^{\prime \prime}$ ) on these sources. Radiative transfer analysis of the line fluxes resulted in calculating high temperatures of $\sim 40000-50000 \mathrm{~K}$. It led Grave et al. (2013) to argue that the spectral types of the embedded stars were O3-O5 (Grave et al. 2013). The temperature estimate is uncertain by up to $5000 \mathrm{~K}$, depending on other factors. Even so, the two sources IRS 1 and IRS 2 can account for at least half of the total bolometric luminosity and most of the 
LyC flux estimated for the G333.6-0.2 UCHII region. We note that Rubin et al. (1994) estimated a temperature of $\sim 36000 \mathrm{~K}$ for this region, even when they used far-infrared spectroscopic data obtained with beam sizes nearly a factor of ten larger than those used by Grave et al. (2013). These data therefore included contributions from other competing ionizing sources in the vicinity. The resulting radiation flux from IRS 1 and IRS 2 is large enough to imitate a light fluid, if appropriately reprocessed and redistributed.

I will present a simplistic analysis of various pressure contributions similar to the analysis carried out by Lopez et al. (2011) to compare it with the simulations of KKM09. Such analysis is useful to study the global energy budget in HII regions, and computing forces on an irradiated medium requires a detailed treatment similar to that of Jacquet \& Krumholz (2011) and Jiang et al. (2013). The choice to nevertheless adopt the method of Lopez et al. (2011) is motivated by the limited data on hand, which lack sufficient measurements on opacity (and its variation), as well as the mass and composition of the material in the region. This analysis is meant only to demonstrate that the radiation pressure supercedes the ionized gas pressure in a general sense. If we assume that both IRS 1 and IRS 2 represent on average $05.5 \mathrm{~V}$ spectral-type objects (a conservative adoption based on the temperature mentioned above), following Vacca et al. (1996), each of these sources should have a luminosity of $L_{*} \sim 4 \times 10^{5} L_{\odot}$. The direct radiation pressure can then be calculated using the equation $P_{\mathrm{dir}}=L / 4 \pi \mathrm{r}^{2} \mathrm{c}$, under the assumption that optical depth $\tau \sim 1$. This pressure is $1.4 \times 10^{-7} \mathrm{~Pa}$ and $3.86 \times 10^{-9} \mathrm{~Pa}$ respectively at distances of $1^{\prime \prime}$ and $6^{\prime \prime}$ (3600 AU and $21000 \mathrm{AU}$ ) from the plane joining the two sources. It is comparable to the ionized gas pressure of $11.5 \times 10^{-8} \mathrm{~Pa}$, which is computed by adopting the electron number density and temperature estimated from $\mathrm{H} 90 \alpha$ observations (Fujiyoshi et al. 2006) and using the equation $P_{\mathrm{HII}} \sim 2 n_{\mathrm{e}} k T_{\mathrm{HII}}$. Because the spatial resolution of the $\mathrm{H} 90 \alpha$ observations is $1.6^{\prime \prime}$, we have used the values $n_{\mathrm{e}}=513000 \mathrm{~cm}^{-3}$ and $T_{\mathrm{HII}}=8350 \mathrm{~K}$, which were estimated using the data closest to the plane of IRS 1 and IRS 2.

The pressure from the X-ray emitting gas, which includes contributions from the stellar winds, was calculated using the equation $P_{\mathrm{X}}=1.9 n_{\mathrm{X}} k T_{\mathrm{X}}$, where $n_{\mathrm{X}}$ and $k T_{\mathrm{X}}$ were obtained from the Chandra X-ray data analysis (see Sect. 2). The resulting pressure is about two to three orders of magnitude lower than the radiation pressure and therefore considered insignificant.

These conservative "back of the envelope" calculations show that in this particular target, the radiation pressure supercedes the ionized gas pressure at least up to a distance of $5000 \mathrm{AU}$ from the plane containing IRS sources. The direct radiation can behave like a light fluid only when the radial isotropy is destroyed; this can happen primarily through turbulence within the outflow bubble and/or the irregular dust envelope evidenced by the $18 \mu \mathrm{m}$ data, which can reprocess and re-radiate through scattering events. The resulting non-isotropic flux escaping through the optically thin ionised outflow bubble is then powerful enough to imitate a lighter fluid upholding the dense overlying fluid, which in this case can be largely ionized.

I note the concentration of features in Figs. 3 and $4 \mathrm{a}$ at about $1^{\prime \prime}$ above the plane of the sources. This is approximately the distance up to which the pressure from the radiation fluid balances the heavier fluid above. When this delicate balance is perturbed, which can occur primarily due to the turbulence within the ionized gas, RTI can develop. In this case, it is visualised by the observed features. The instabilities result in dense structures, which are then pulled by the gravitational force towards the disk plane. The two linear features with finger/hook morphology in
Figs. 3 and 4a, which appear connected to the IRS sources, then represent such pulled-back structures.

In this simplistic scenario, the radiation pressure supercedes gravitational pressure beyond a certain distance from the star and equals that of ionized gas pressure (about $5000 \mathrm{AU}$ in this case), which can explain the balancing zone by the lighter radiation fluid of the overlying dense material fluid. It should be noted that in the treatment of generalized Eddington limit, such effects would not be possible because both the radiation and gravitational forces scale as $r^{-2}$. It is expected to occur only when a high light-to-mass ratio $(L / M \sim 2500)$, driven by Kelvin-Helmholtz contraction, is sustained during the early stages. The requirement here is that the radiation be dissipated in a preferential direction, as discussed in KKM09. In this particular target, IRS 1 and IRS 2 have multiple characteristics of representing single massive stars, as discussed by Grave et al. (2013). The hypothesis is based on the statistically rare case of tight, very massive equalmass binaries with separations of less than 500 AU (for e.g. Sana et al. 2012). When the mass, luminosity, and multiplicity of the two IRS sources are ascertained, it will be possible to accurately compute the pressure balance using future observations.

Additionally, in the scenario of KKM09, the infalling gas strikes the walls of the radiation-driven bubble, which will slide back along the walls to the mid-plane. The two outermost vertical structures seen in Fig. 4a (marked using orange lines) can represent such walls of the radiation bubble, curiously, no dense features are observed beyond these walls.

\section{Discussion}

The density structures discussed above, which are satisfying signatures of RTI, should be overdensities of ionized gas as it is completely embedded inside the observed compact HII region. The conjecture made in the previous section that the observed features are not due to hot gas expanding at the sound speed of $\sim 10 \mathrm{~km} \mathrm{~s}^{-1}$ is supported by the observed H90 $\alpha$ data (Fujiyoshi et al. 2006). Of the $36 \mathrm{H} 90 \alpha$ profiles obtained to map this target, six are clearly double peaked; the two peaks are found on either side of $V_{\mathrm{LSR}}$, and have an average peak-to-peak velocity shift of $30 \mathrm{~km} \mathrm{~s}^{-1}$. These double-peaked profiles closely overlap and surround the region containing the newly discovered density features. In Fig. 4a, four of the most prominent profiles are overplotted on the adaptive optics image, which can be seen to surround the sources IRS 1 and IRS 2. This overlay allows an ad hoc interpretation of the previous $\mathrm{H} 90 \alpha$ data, proving which demands higher resolution radio data: a) profiles along the eastwest direction originate in an expanding outflow bubble (the two peaks are due to the two walls of the bubble) and b) profiles along the north-south direction represent some rotation involving two components.

The bipolar nebula separated by a dark lane (as depicted by Fig. 1) is an indication that the system is viewed at a small inclination angle in the sky-plane, with the disk/envelope appearing nearly edge-on. As the outflow axis is nearly aligned in the plane of the sky, the two peaks (blue and red shifted) of the double-peaked profiles represent the average motion of matter moving toward and away from us within the expanding bubble. The line-of-sight velocity (peak-to-peak difference) depicted by H90 $\alpha$ profiles \#8 and \#14 (see Fujiyoshi et al. 2006) of $20 \mathrm{~km} \mathrm{~s}^{-1}$ and $24 \mathrm{~km} \mathrm{~s}^{-1}$ correspond to these components in the two outflow lobes.

The profiles \#7 and \#15 which roughly enclose the two edges of the observed infrared dark lane, can represent rotation of a 
large ( $\sim 5000 \mathrm{AU})$ toroid. The double peaks and the shift in the heights of blue and red peaks arising from opposite sides can be explained only if this rotating structure includes two individual structures rotating in the same direction and viewed edge-on. Such a scenario strikingly resembles the simulations by KKM09. Given that the $\mathrm{H} 90 \alpha$ observations are obtained at a spatial resolution of 1.6", motions at smaller scales are mixed up and averaged out, resulting in a double peak at the two edges. I note that Fujiyoshi et al. (2006) considered the hypothesis of rotation and rejected it to propose blister/champagne flow to explain the double-peaked profiles. This interpretation was understandably influenced by the uncertainty that the heart of G333.6-0.2 hosts a cluster of $\mathrm{O} 7-\mathrm{O} 8$ ionizing sources. The new observations here and in Grave et al. (2013) pinpoint IRS 1 and IRS 2 as the main luminous sources resulting in the high $\mathrm{L} / \mathrm{M}$ condition necessary for the proposed scenario. Indeed, the proposed outflow interpretation of Fujiyoshi et al. (2006) can now be associated directly with IRS 1. In hindsight, it also appears that the dark lane visible in the near-infrared images may not be a toroid after all. The real toroid or disks must coincide with the IRS sources and the $18 \mu \mathrm{m}$ emission contours, while the dark patch marked as a possible toroid in Fig. 1 is actually a result of low-density cavity material. This can be visualised by direct comparison of Figs. $4 \mathrm{a}$ and $4 \mathrm{~b}$.

Essentially all features shown in Fig. 4a, namely the disk/toroid and the outflow cavity encompassing the observed sources, are immersed and depict details of the compact HII region. If the above ad hoc interpretation of the H90 $\alpha$ doublepeaked profile is proven to be valid, the sources IRS 1 and IRS 2 are likely to be accreting ionized gas (rather than molecular material), as proposed by Keto $(2002,2003)$. In fact, $\mathrm{H}_{2}$ emission is found to be insignificant in this region (Moneti \& Moorwood 1989; Fujiyoshi et al. 2006), arguing for the lack of any molecular gas.

If the observed compact HII region (Fujiyoshi et al. 2006) in this target $\left(\sim 6^{\prime \prime} \sim 20000 \mathrm{AU}\right)$ represents the radiation-driven bubble, then it appears to be much larger than the 3000 AU bubble from simulations by KKM09. It is possible that the observed 6" size of the compact HII region has significant contributions from other competing sources in the vicinity. Also, the sizescale of RTI predicted by KKM09 is a factor of five to ten smaller than those discovered here. The uncertainties inherent to both observations and theory impede a quantitative comparison at this stage. Next, the KKM09 simulations did not consider the influence of factors such as direct radiation, magnetic fields, and photoionisation, which can impact the size scales of the expected features. A detailed discussion on the effects of various caveats in producing RTI is presented by Krumholz \& Thompson (2012). While many arguments are used here to discuss the less likely role of such other factors, ruling out their contribution against RTI can be challenging, especially because simulations including them also resulted in producing fine filamentary features (Peters et al. 2011). A multitude of new high-spatial resolution measurements evaluating the composition, opacity, mass, luminosity etc., should be quantitatively compared to the simulation outcomes (Jacquet \& Krumholz 2011; Jiang et al. 2013; Krumholz \& Thompson 2012) before confirming the crucial role of RTI in the mechanism of high-mass star formation. The observations and analysis presented here nevertheless show that RTI may indeed play an important role in the formation of massive stars and observational signatures at physical scale resolutions of a thousand AU or less will require careful attention in the future to confront theoretical scenarios.

Acknowledgements. I am grateful to Mark Krumholz for inspiring and educative discussions of remarkable clarity, which enlightened me with the essentials of some theoretical investigations, that had been beyond my grasp by solely reading the literature. This work greatly benefitted from the objective criticism and input from anonymous referees, both for the present manuscript and from earlier submissions to Nature and Science journals. Warm thanks are due to A.R. Rao of TIFR, Mumbai for his advice on the X-ray data analysis and to outreach team at CAUP for help with Fig. 3. Part of this work was carried out under the auspices of an EU FP7 Marie-Curie IRSES-230843 programme. I am supported by a Ciência 2007 contract, funded by FCT (Portugal) and POPH/FSE (EC). This work has made use of ESO archival services, Spitzer, and Chandra Data Archives.

\section{References}

Adams, F. C., Ruden, P., \& Shu, F. H. 1989, ApJ, 347, 959

Arzoumanian, D., Andre, Ph., Didelon, P., et al. 2011, A\&A, 529, A6

Becklin, E. E., Frogel, J. A., Neugebauer, G., Persson, S. E., \& Wynn-Williams, C. G. 1973, ApJ, 182, 125

Cunningham, A. J., Klein, R. I., Krumholz, M. R., \& McKee, C. F. 2011, ApJ, 740,107

Feldt, M., Puga, E., Lenzen, R., et al. 2003, ApJ, 599, L91

Felli, M., Massi, M., Staude, H. J., et al. 1984, A\&A, 135, 261

Fujiyoshi, T., Smith, C. H., Moore, T. J. T., et al. 1998, MNRAS, 296, 225 Fujiyoshi, T., Smith, C. H., Wright, C. M., et al. 2001, MNRAS, 327, 233 Fujiyoshi, T., Smith, C. H., Moore, T. J. T., et al. 2005, MNRAS, 356, 801 Fujiyoshi, T., Smith, C. H., Caswell, J. L., et al. 2006, MNRAS, 368, 1843 Furuya, R. S., Kitamura, Y., Wootten, A., Claussen, M. J., \& Kawabe, R. 2002, in Cosmic Masers: From Proto-Stars to Black Holes, IAU Symp. Proc., eds. V. Mineese, \& M. Reid, ASP, 206, 22

Grave, J. M. C., Kumar, M. S. N., Ojha, D. K., Teixeira, G. D. C., \& Pace, G. 2013, A\&A, submitted [arXiv: 1307. 0566]

Hennebelle, P., Commerçon, B., Joos, M., Klessen, R. S., Krumholz, M. R., et al. 2011, A\&A, 528, A72

Jacquet, E., \& Krumholz, M. R. 2011, ApJ, 730, 116

Jiang, Y.-F., Davis, S. W., \& Stone, J. M. 2013, ApJ, 763, 102

Keto, E. R. 2002, ApJ, 568, 754

Keto, E. R. 2003, ApJ, 599, 1196

Krumholz, M. R., \& Thompson, T. A. 2012, ApJ, 760, 155

Krumholz, M. R., Klein, R. I. McKee, C. F., Offner, S. S. R., \& Cunningham, A. J. 2009, Science, 323, 754

Kuiper, R., Hubert, K., Beuther, H., \& Henning, T. 2010, ApJ, 722, 1556

Kuiper, R., Hubert, K., Beuther, H., \& Henning, T. 2011, ApJ, 732, 1

Kuiper, R., Hubert, K., Beuther, H., \& Henning, T. 2012, ApJ, 537, A122

Kurtz, S. E., Churchwell, E., \& Wood, D. O. S. 1994, ApJS, 91, 659

Lo, N., Cunningham, M. R., Jones, P. A., et al. 2009, MNRAS, 395, 1021

Lopez, L. R., Krumholz, M. R., Bolatto, A. D., Prochaska, J. X., \& RamirezRuiz, A. 2011, ApJ, 731, 91

McGee, R. X., Newton, L. M., Batchelor, R. A. 1975, Aust. J. Phys., 28, 185

Minniti, D., Lucas, P. W., Emerson, J. P., et al. 2010, New Astron., 15, 433

Moneti, A., \& Moorwood, A. F. M. 1989, in Infrared Spectroscopy in Astronomy, ESA, 299

Mookerjea, B., Kramer, C., Nielbock, M., \& Nyman, L. A. 2004, A\&A, 426, 119

Myers, A. T., McKee, C. F., Cunningham, A. J., Klein, R. I., \& Krumholz, M. R. 2013, ApJ, 766, 97

Nakano, T. 1989, ApJ, 345, 464

Natta, A., \& Oliva, E. 1985, A\&A, 152, 300

Peters, T., Banerjee, R., Klessen, R. S., et al. 2010a, ApJ, 711, 1017

Peters, T., Mac Low, M.-M., Banerjee, R., et al. 2010b, ApJ, 719, 831

Peters, T., Banerjee, R., Klessen, R. S., et al. 2011, ApJ, 729, 72

Rubin, R. H., Simpson, J. P., Lord, S. D., et al. 1994, ApJ, 420, 772

Sana, H., de Mink, S. E., de Koter, A., et al. 2012, Science, 337, 444

Sutton, E., Becklin, E. E., \& Neugebauer, G. 1974, ApJ, 190, 69

Urquhart, J. S., Hoare, M. G., Purcell, C. R., et al. 2009, A\&A, 501, 539

Vacca, W. D., Garmany, C. D., \& Shull, J. M. 1996, ApJ, 460, 914

Wood, D. O. S., \& Churchwell, E. 1989, ApJS, 69, 831

Yorke, H. W., \& Sonnhalter, C. 2002, ApJ, 569, 846

Zinnecker, H., \& Yorke, H. W. 2007, ARA\&A, 45, 481 